\title{
Symmetric Stability in Symmetric Games
}

\author{
Andreas Hefti \\ School of Management and Law, University of Zurich, Winterthur, Switzerland \\ Email: heft@zhaw.ch \\ Received 29 March 2016; accepted 6 June 2016; published 9 June 2016 \\ Copyright (C) 2016 by author and Scientific Research Publishing Inc. \\ This work is licensed under the Creative Commons Attribution International License (CC BY). \\ http://creativecommons.org/licenses/by/4.0/ \\ c) (i) Open Access
}

\begin{abstract}
The idea of symmetric stability of symmetric equilibria is introduced which is relevant, e.g., for the comparative-statics of symmetric equilibria with symmetric shocks. I show that symmetric stability can be expressed in a two-player reduced-form version of the $N$-player game, derive an elementary relation between symmetric stability and the existence of exactly one symmetric equilibrium, and apply symmetric stability to a two-dimensional $N$-player contest.
\end{abstract}

\section{Keywords}

\section{Symmetric Games, Symmetric Equilibrium, Stability, Uniqueness}

\section{Introduction}

In this note I develop the idea of symmetric stability of symmetric equilibria in symmetric $N$-player games. With symmetric equilibria it is reasonable to consider dynamics where the set of trajectories is restricted by symmetric initial conditions. This is particularly relevant when studying the comparative-statics of symmetric equilibria to a common shock, such as changing the prize in a contest or a tax parameter in the Cournot model, since this has symmetric effects on symmetric players both in terms of the initial displacement and the subsequent adjustment process. Symmetric stability conditions can be expressed in terms of a best-reply function obtained by fixing the strategies of all other players to the same action. Given a $k$-dimensional strategy space, this reduces the dimensionality of the stability problem from $N k$ to $k$, while retaining all relevant information about symmetric equilibria and their symmetric stability. By means of this reduced form I prove that the existence of a single symmetric equilibrium is the same formal property as global symmetric stability in regular one-dimensional games, independent of the number of players. Further, stability under symmetric adjustments implies the existence of only one symmetric equilibrium for any finite-dimensional strategy space, and symmetric stability provides a meaningful restriction for the possible comparative-static patterns of symmetric equilibria. All results are independent of the possible existence of asymmetric equilibria, and the practical usefulness of symmetric stability is briefly illustrated by means of a two-dimensional $N$-player contest. 


\section{Symmetric Games}

I consider games of $N \geq 2$ players. $x_{g} \equiv\left(x_{g 1}, \cdots, x_{g k}\right) \in S(k)$ is a strategy of player $g$, where $S \equiv S(k)=x_{i=1}^{k} S_{i}$ with $S_{i}=\left[0, \bar{S}_{i}\right] \subset \mathbb{R}, \bar{S}_{i}>0$, and interior $\operatorname{Int}\left(S_{i}\right)$. All players have identical strategy space. The payoff of $g$ is represented by a $C^{2}$ function $\Pi^{g}\left(x_{1}, \cdots, x_{g}, \cdots, x_{N}\right) \equiv \Pi\left(x_{g}, x_{-g}\right)$, which is strongly quasiconcave ${ }^{1}$ in $x_{g}$. In a symmetric game all players have identical payoff function in the sense that

$$
\Pi^{g}\left(x_{1}, \cdots, x_{g}, \cdots, x_{N}\right)=\Pi^{\sigma(g)}\left(x_{\sigma(1)}, \cdots, x_{\sigma(g)}, \cdots, x_{\sigma(N)}\right)
$$

for any permutation $\sigma$ of $\{1, \cdots, N\}$. With the above assumptions, player $g$ 's best-reply function $\varphi\left(x_{-g}\right) \equiv \varphi^{g}\left(x_{-g}\right)$ is continuous, and differentiable at interior points. Given the focus on symmetric equilibria one can restrict attention to a reduced-form problem by picking an indicative player $(g=1)$, and requiring all opponents to play the same strategies, i.e. $\bar{x}_{-1}=(\bar{x}, \cdots, \bar{x}), \bar{x} \in S$. Let $\bar{\Pi}\left(x_{1}, \bar{x}\right) \equiv \Pi\left(x_{1}, \bar{x}_{-1}\right)$ with corresponding best-reply $\tilde{\varphi}(\bar{x}) \equiv \varphi\left(\bar{x}_{-1}\right)$ and derivative $\partial \tilde{\varphi}(\bar{x})$. Any symmetric equilibrium $x^{*}$ is identifiable by its first projection $x_{1}^{*} \in S, x^{*}$ is a symmetric equilibrium if $x_{1}^{*}=\tilde{\varphi}\left(x_{1}^{*}\right)$, a symmetric equilibrium always exists and the set of symmetric equilibria is compact [1].

\subsection{Symmetric Stability}

I mostly restrict attention to the system of gradient dynamics ${ }^{2}$

$$
\dot{x}_{j}=S \nabla_{j} \Pi^{j}(x), \quad 1 \leq j \leq N
$$

where $S$ is a $k \times k$ positive-diagonal adjustment matrix, and $\nabla_{j} \Pi^{j}(x)=\frac{\partial \Pi^{j}(x)}{\partial x_{j}}$. A solution to (1) has the form $x(t)=\left(x_{j}(t)\right)_{1<j<N}$, where $x_{j}(t)=\left(x_{j 1}(t), \cdots, x_{j k}(t)\right)$ is the trajectory of $j$. I consider a restricted version of this trajectory map, where initial values $x(0)$ are symmetric, i.e. $x_{1}(0)=\cdots=x_{N}(0)$. Then, by symmetry, $x_{j}(t)$ is the same for all players and solves

$$
\dot{x}_{1}=S \nabla \tilde{\Pi}\left(x_{1}\right), \quad \text { where }\left.\quad \nabla \tilde{\Pi}\left(x_{1}\right) \equiv \frac{\partial \bar{\Pi}\left(x_{1}, \bar{x}\right)}{\partial x_{1}}\right|_{\bar{x}=x_{1}}
$$

I say that an interior symmetric equilibrium $x^{*}$ is symmetrically stable if the dynamics induced by (2) converge to $x^{*}$ whenever $x(0)$ is close to $x^{*} .{ }^{3}$ Hence:

Definition 1 (Symmetric stability) The symmetric equilibrium $x^{*} \in \operatorname{Int}(S)$ is symmetrically stable if all eigenvalues of $\hat{J}\left(x_{1}^{*}\right)$, the Jacobian corresponding to (2), have negative real parts.

$x^{*}$ is symmetrically unstable if at least one eigenvalue of $\hat{J}\left(x_{1}^{*}\right)$ has positive real part. Stability of (1) implies symmetric stability, but not vice-versa (see Figure 1). Let $C r^{s}=\left\{x_{1} \in S: \nabla \tilde{\Pi}\left(x_{1}\right)=0\right\}$, and note that $\nabla \tilde{\Pi}\left(x_{1}\right): S \rightarrow \mathbb{R}^{k}, \quad x_{1} \mapsto \nabla \tilde{\Pi}\left(x_{1}\right)$ is a $C^{1}$-vector field with $k \times k$ Jacobian $\tilde{J}\left(x_{1}\right)$. A symmetric game is (symmetrically) regular if i) $\nabla \tilde{\Pi}$ has only regular zeroes ${ }^{4}$ and ii) $\nabla \tilde{\Pi}$ points inwards at the boundary of $S$. Any future reference to a regular game means "symmetrically" regular, which is a weaker condition than general regularity of a symmetric game (see [2]). The first theorem below reveals the general connection between symmetric stability and the existence of a single symmetric equilibrium, depending on the dimensionality of the strategy space. Its proof exploits an essential relation between $\hat{J}, \tilde{J}$ and $\tilde{\varphi}$.

Lemma 1 For $x_{1} \in \operatorname{Int}(S)$ :

$$
\hat{J}\left(x_{1}\right)=S \tilde{J}\left(x_{1}\right)=S\left(I-\partial \tilde{\varphi}\left(x_{1}\right)\right)
$$

${ }^{1}$ Meaning: $\quad z \cdot z=1, \quad z \cdot \frac{\partial \Pi^{g}(x)}{\partial x_{g}}=0 \Rightarrow z \cdot \frac{\partial^{2} \Pi^{g}(x)}{\partial x_{g} \partial x_{g}} z<0 \quad$ (see [1]).

${ }^{2}$ This is a standard dynamics, see e.g. [3]-[5].

${ }^{3}$ Equivalently, $x^{*}$ is symmetrically stable if the dynamics (1) converge to $x^{*}$ for any symmetric starting point close enough to $x^{*}$.

${ }^{4} x_{1} \in C r^{s} \Rightarrow \operatorname{Det}\left(\tilde{J}\left(x_{1}\right)\right) \neq 0$. 

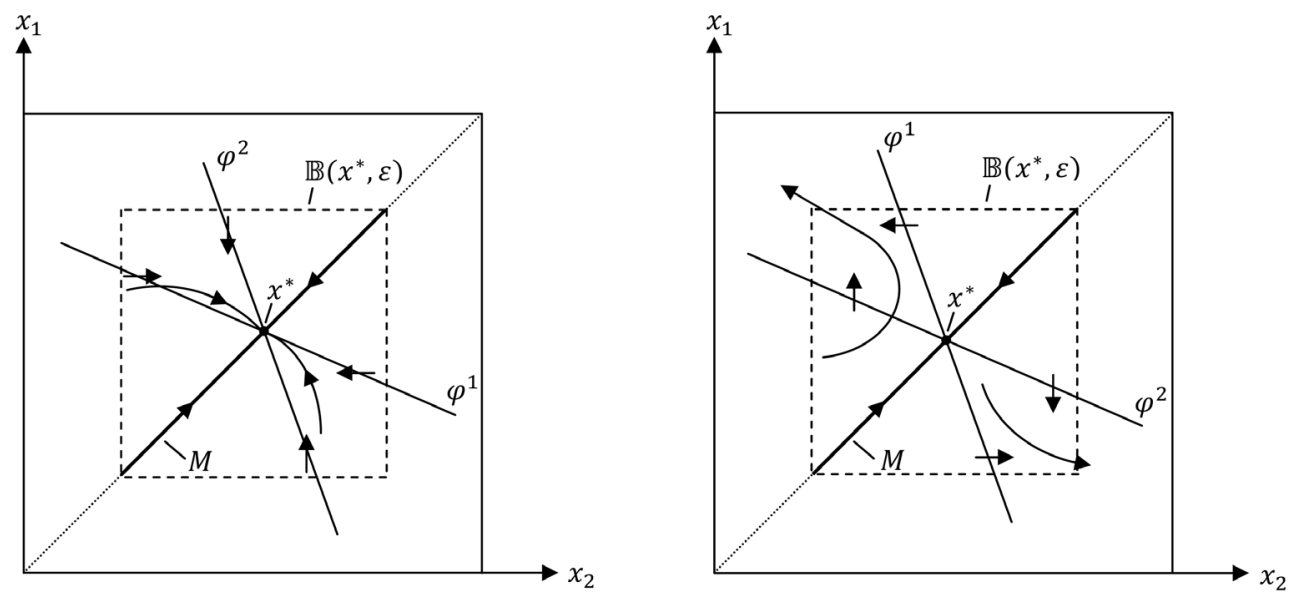

Figure 1. Stable (left) and only symmetrically stable (right).

Proof: The first equality is immediate. Next, decompose $\tilde{J}\left(x_{1}\right)=\left.\frac{\partial \nabla \tilde{\Pi}\left(x_{1}, \bar{x}\right)}{\partial x_{1}}\right|_{\bar{x}=x_{1}}+\left.\frac{\partial \nabla \tilde{\Pi}\left(x_{1}, \bar{x}\right)}{\partial \bar{x}}\right|_{\bar{x}=x_{1}}$. By the Implicit Function Theorem (IFT) $\partial \tilde{\varphi}\left(x_{1}\right)=\left.\left(\left.\frac{\partial \nabla \tilde{\Pi}\left(x_{1}, \bar{x}\right)}{\partial x_{1}}\right|_{\bar{x}=x_{1}}\right)^{-1} \frac{\partial \nabla \tilde{\Pi}\left(x_{1}, \bar{x}\right)}{\partial \bar{x}}\right|_{\bar{x}=x_{1}}$ which, together with the decomposition of $\tilde{J}$ gives the second equality.

Let $\tilde{\Pi}_{\alpha \beta}\left(x_{1}\right) \equiv \frac{\partial \nabla_{\alpha} \tilde{\Pi}\left(x_{1}\right)}{\partial x_{1 \beta}}, 1 \leq \alpha, \beta \leq k$, where $\nabla_{a} \tilde{\Pi}$ is the $\alpha$-th projection of $\nabla \tilde{\Pi}$.

Theorem 1 (i) If $k=1$ then $x^{*}$ is a symmetrically stable equilibrium if $\tilde{\Pi}_{11}\left(x_{1}^{*}\right)<0$ or equivalently if $\tilde{\varphi}^{\prime}\left(x_{1}^{*}\right)<1$.

(ii) For $k=2$ a symmetric equilibrium $x^{*}$ is symmetrically stable if $-\tilde{J}\left(x_{1}^{*}\right)$ has only positive principal minors.

(iii) If $k \geq 1$ and a regular games has multiple symmetric equilibria, then there is at least one symmetrically unstable equilibrium.

Proof: (i) Follows from lemma 1. (ii) Let $\lambda_{1}, \lambda_{2}$ be the eigenvalues of $\hat{J}\left(x_{1}^{*}\right)$, and $s_{1}, s_{2}>0$ be the diagonal entries of $S$. Lemma 1 and the condition in (ii) imply $\lambda_{1}+\lambda_{2}=\operatorname{Trace}\left(\hat{J}\left(x_{1}^{*}\right)\right)=s_{1} \tilde{\Pi}_{11}\left(x_{1}^{*}\right)+s_{2} \tilde{\Pi}_{22}\left(x_{1}^{*}\right)<0$ and $\lambda_{1} \lambda_{2}=\operatorname{Det}\left(\hat{J}\left(x_{1}^{*}\right)\right)=s_{1} s_{2} \operatorname{Det}\left(\tilde{J}\left(x_{1}\right)\right)>0$. Hence $\lambda_{1}, \lambda_{2}$ have negative real parts. (iii) Follows from applying the Index theorem to the symmetric gradient field $\nabla \tilde{\Pi} .^{5}$ The index of a zero of $\nabla \tilde{\Pi}$ is +1 if $\operatorname{Det}\left(-\tilde{J}\left(x_{1}\right)\right)>0$ and -1 if $\operatorname{Det}\left(-\tilde{J}\left(x_{1}\right)\right)<0$. With multiple symmetric equilibria a symmetric equilibrium with -1 index exists, and $\operatorname{Det}\left(-\tilde{J}\left(x_{1}^{*}\right)\right)<0$. By lemma $1 \operatorname{Det}\left(-\hat{J}\left(x_{1}\right)\right)=s_{1} \cdots s_{k} \cdot \operatorname{Det}\left(-\tilde{J}\left(x_{1}\right)\right)$, hence $\operatorname{Det}\left(-\hat{J}\left(x_{1}^{*}\right)\right)<0$, which implies existence of at least one eigenvalue with negative real part.

It follows from (iii) that if each $x_{1} \in C r^{s}$ verifies symmetric stability then exactly one symmetric equilibrium exists. ${ }^{6}$ In the one-dimensional case an even stronger relation between symmetric stability and the number of symmetric equilibria applies: ${ }^{7}$

Corollary 1 Let $k=1$ in a regular game. There exists an odd number of symmetrically stable equilibria.

\section{${ }^{5}$ See [5].}

${ }^{6}$ If all eigenvalues of $\hat{J}\left(x_{1}\right)$ have negative real parts, then $\operatorname{Det}\left(-\hat{J}\left(x_{1}\right)\right)>0$ and by (3) also $\operatorname{Det}\left(-\tilde{J}\left(x_{1}\right)\right)>0$. Thus every $x_{1} \in C r^{s}$ has index +1 , which by the index theorem implies existence of a unique symmetric equilibrium. This type of relation between the index of certain vector fields and stability conditions is known in other settings (see [6] and the references therein).

${ }^{7}$ The claims in corollary 1 are generally restricted to $k=1$. 
Moreover, a symmetric equilibrium is globally symmetrically stable if $x^{*}$ is the only symmetric equilibrium.

Proof: Given regularity, a zero of $\nabla \tilde{\Pi}\left(x_{1}^{*}\right)$ is symmetrically stable if $\tilde{\varphi}^{\prime}\left(x_{1}^{*}\right)<1$. Hence symmetrically stable equilibria have index +1 and the first claim follows from the Index theorem. Necessity in the second claim is trivial. For sufficiency, note that uniqueness of $x_{1}^{*}$ implies $\tilde{J}\left(x_{1}^{*}\right)<0$ (the corresponding index is +1 ). From (3) deduce that $\tilde{\varphi}^{\prime}\left(x_{1}^{*}\right)<1$. Hence $\tilde{\varphi}(\bar{x})$ crosses the $45^{\circ}$-line from above. Thus $\tilde{\varphi}(\bar{x})>\bar{x}$ whenever $\bar{x}<x_{1}^{*}$. Let $\bar{x}<x_{1}^{*}$. Because $\nabla \bar{\Pi}(\tilde{\varphi}(\bar{x}), \bar{x})=0$ it follows from $\tilde{\varphi}(\bar{x})>\bar{x}$ and strong quasicon-cavity that $\nabla \tilde{\Pi}(\bar{x})=\nabla \bar{\Pi}(\bar{x}, \bar{x})>0$. Hence $\dot{x}_{1}(t)>0$ whenever $x_{1}(t)<x_{1}^{*}$ (Figure 2). Similarly, $\dot{x}_{1}(t)<0$ whenever $x_{1}(t)>x_{1}^{*}$.

It may be noted from the above proof (or Figure 2) that the second claim of corollary 1 remains valid if there is a single symmetric equilibrium which belongs to the boundary $\left(x_{1}^{*} \in \partial S\right)$.

Best-reply dynamics. Another standard dynamics in the literature are dynamics defined directly over the best-reply functions. ${ }^{8}$ These dynamics are of the form

$$
\dot{x}_{j}=S\left(\varphi\left(x_{-j}\right)-x_{j}\right), \quad 1 \leq j \leq N
$$

and the symmetric restriction analogously to (2) yields

$$
\dot{x}_{1}=S\left(\tilde{\varphi}(\bar{x})-x_{1}\right)
$$

A symmetric equilibrium $x^{*}$ is symmetrically stable with respect to (4) if the Jacobian, $\breve{J}\left(x_{1}\right)=-S\left(I-\tilde{\varphi}\left(x_{1}\right)\right)$ has only eigenvalues with negative real parts. It follows that corollary 1 and theorem 1 (i) and (iii) apply, without modification, to the dynamics (4). The latter follows from (3) and the proof of theorem 1, and the former can be deduced directly from (4) together with Figure $2 .^{9}$

Relation to comparative statics. Typically, the IFT is the main formal tool to (locally) sign the comparativestatic effects. ${ }^{10}$ Stability conditions allow to robustly sign comparative-static effects [3] and additionally assure local convergence after a small shock, which many deem a natural requirement of a comparative-static predicttion. A symmetrically unstable equilibrium is not re-established after a symmetric shock. Moreover, symmetri-

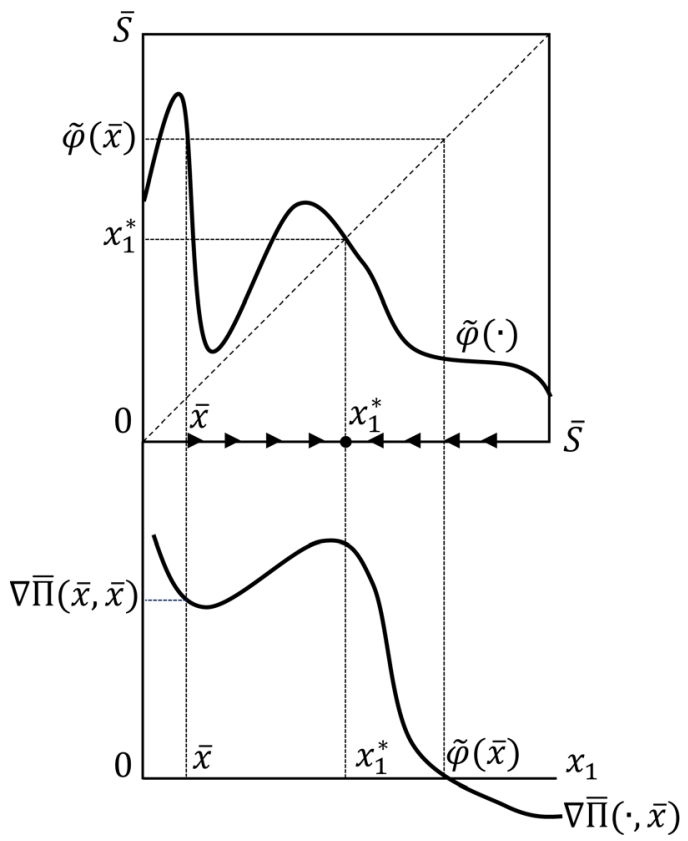

Figure 2. Corollary 1.

${ }^{8}$ See e.g. [5] [7]-[9].

${ }^{9}$ It can be noted occasionally from the figure that strong quasiconcavity is generally indispensable for corollary 1 to apply with the gradient dynamics, but not with (4).

${ }^{10} \mathrm{~A}$ well-known exception is supermodular games (see [5]). 
cally unstable equilibria may "pervert" the comparative-statics. To illustrate consider a regular game with three symmetric equilibria $x^{A}(c), x^{B}(c), x^{C}(c)$ (see Figure 3 ) where $c$ is an exogenous common parameter. $A$ and $B$ are symmetrically stable (index +1 ), but $C$ is symmetrically unstable (index -1 ). Consider a symmetric parameter shift $c \rightarrow c^{\prime}$ and assume that $\tilde{\varphi}\left(\bar{x}, c^{\prime}\right)>\tilde{\varphi}(\bar{x}, c)$. As is suggested by the figure (formally apply the IFT) points $A$ and $B$ both increase to $A^{\prime}$ and $B^{\prime}$. As both $A^{\prime}$ and $B^{\prime}$ are symmetrically stable, the symmetric dynamics (2) converge from $A$ to $A^{\prime}$ or from $B$ to $B^{\prime}$, consistent with the suggested shift of $\tilde{\varphi}$. For the symmetrically unstable point $C$ we see that $C^{\prime}<C$ (a consequence of the negative index), contradicting the direction suggested by $\tilde{\varphi}\left(\bar{x}, c^{\prime}\right)>\tilde{\varphi}(\bar{x}, c)$. As $C$ lies in the basin of attraction of $B^{\prime}$ the dynamics do not move down to $C^{\prime}$ but monotonically up to $B^{\prime}$ (which is also inconsistent with "small" changes). Hence the comparative-statics suggested by the IFT and the dynamics disagree at the unstable equilibria, and the IFTprediction $C \rightarrow C^{\prime}$ could never be supported as a stable equilibrium.

\subsection{Application: Two-Dimensional Contest with Endogenous Price}

To illustrate symmetric stability in an example consider a payoff of the form

$$
\Pi(f, p)=\pi(f, \bar{f}) V(p, \bar{p})-C(f)
$$

The interpretation is that $N$ contestants choose their strategies, the pairs $\left(f_{j}, p_{j}\right)$, to obtain a prize worth $V(\cdot)$, where the value of a prize is endogenously determined. A specific context is provided by [10], where firms compete in salience and prices for attention-constrained consumers. ${ }^{11}$ Assume that $C^{\prime}(f)>0, C^{\prime \prime}(f) \leq 0$ and $\pi(f, f)>0$ (everybody has a chance to seize a prize) for $f>0$, and $V(p, p)>0$ (a prize is worthwhile seizing) for $p>0$. An interior symmetric equilibrium $(p, f)>0$ solves

$$
\begin{aligned}
& \pi(f, f) V_{1}(p, p)=0 \\
& \pi_{1}(f, f) V(p, p)-C^{\prime}(f)=0
\end{aligned}
$$

with associated Jacobian

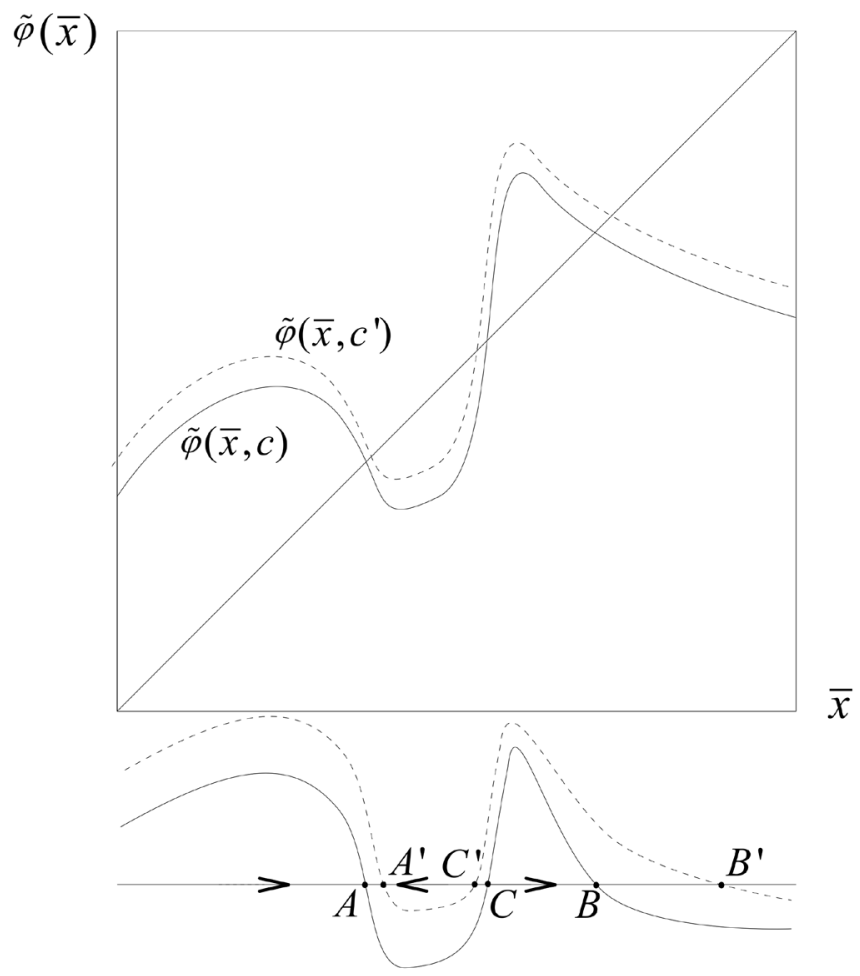

Figure 3. Symmetric stability and comparative statics.

\footnotetext{
${ }^{11}$ Payoff (5) is compatible with multiple prizes.
} 


$$
\tilde{J}(p, f)=\left(\begin{array}{ll}
\tilde{\Pi}_{p p} & \tilde{\Pi}_{p f} \\
\tilde{\Pi}_{p f} & \tilde{\Pi}_{f f}
\end{array}\right)=\left(\begin{array}{lc}
\pi(f, f) \frac{\partial V_{1}(p, p)}{\partial p} & 0 \\
\pi_{1}(f, f) \frac{\partial V(p, p)}{\partial p} & \frac{\partial \pi_{1}(f, f)}{\partial f} V(p, p)-C^{\prime \prime}(f)
\end{array}\right)
$$

It easily follows from theorem 1 (ii) that $(p, f)$ is symmetrically stable if both $\tilde{\Pi}_{p p}, \tilde{\Pi}_{f f}<0$. By contrast, with dynamics (1) we would need to evaluate the eigenvalues of a $N k \times N k$ matrix. The symmetric stability condition states that second-order direct effects of each own strategy $p, f$ (which must be negative by strong quasiconcavity) are not reversed by the second-order effects of $(\bar{p}, \bar{f})$, a property which is typically satisfied in standard functional examples (see [7]). Moreover, it follows from theorem 1 (iii) that if any $(p, f) \in \mathrm{Cr}^{s}$ verifies this condition and the game is symmetrically regular, a single symmetric and symmetrically stable equilibrium exists.

\section{Acknowledgements}

I thank Diethard Klatte and participants at seminars at University of Zurich, Harvard University and at the UECE Lisbon Game Theory meeting for valuable comments, and Ines Brunner for ongoing support.

\section{References}

[1] Avriel, M., Diewert, W.E. and Zang, I. (1981) Nine Kinds of Quasiconcavity and Concavity. Journal of Economic Theory, 25, 397-420. http://dx.doi.org/10.1016/0022-0531(81)90039-9

[2] Hefti, A. (2014) Equilibria in Symmetric Games: Theory and Applications. ECON Working Paper.

[3] Dixit, A. (1986) Comparative Statics for Oligopoly. International Economic Review, 27, 107-122. http://dx.doi.org/10.2307/2526609

[4] Okuguchi, K. and Yamazaki, T. (2008) Global Stability of Unique Nash Equilibrium in Cournotoligopoly and RentSeeking Game. Journal of Economic Dynamics and Control, 32, 1204-1211. http://dx.doi.org/10.1016/j.jedc.2007.05.003

[5] Vives, X. (1999) Oligopoly Pricing. Cambridge MIT Press, Cambridge.

[6] Hefti, A. (2016) On the Relationship between Uniqueness and Stability in Sum-Aggregative, Symmetric and General Differentiable Games. Mathematical Social Sciences, 80, 83-96. http://dx.doi.org/10.1016/j.mathsocsci.2016.02.008

[7] Hahn, F. (1962) The Stability of the Cournot Oligopoly Solution. The Review of Economic Studies, 29, 329-331. http://dx.doi.org/10.2307/2296310

[8] Al Nowaihi, A. and Levine, P.L. (1985) The Stability of the Cournot Oligopoly Model: A Reassessment. Journal of Economic Theory, 35, 307-321. http://dx.doi.org/10.1016/0022-0531(85)90046-8

[9] Dindos, M. and Mezzetti, C. (2006) Better-Reply Dynamics and Global Convergence to Nash Equilibriumin Aggregative Games. Games and Economic Behavior, 54, 261-292. http://dx.doi.org/10.1016/j.geb.2004.12.001

[10] Hefti, A. (2015) Limited Attention, Competition and Welfare. Working Paper. 